01;07

\title{
Внутризонное поглощение излучения свободными дырками в квантовых ямах GaAs/InGaAs с учетом несферичности $\boldsymbol{k P}$-гамильтониана
}

\author{
(C) Н.В. Павлов, Г.Г. Зегря
}

Физико-технический институт им. А.Ф. Иоффре РАН, Санкт-Петербург, Россия

E-mail: pavlovnv@mail.ru

Поступило в Редакцию 26 июля 2018г.

В окончательной редакции 18 февраля 2019 г.

Принято к публикации 22 февраля 2019г.

\begin{abstract}
Выведена и решена система кейновских уравнений с учетом упругих напряжений и несферичности $k P$-гамильтониана, получены аналитические выражения энергетических спектров носителей заряда. Выполнен расчет коэффициента поглощения света тяжелыми дырками с переходом в спин-отщепленную зону в квантовых ямах $\mathrm{GaAs} / \mathrm{InGaAs}$ для различных направлений поляризации падающего излучения. Показано, что для гетероструктуры GaAs/InGaAs максимальное поглощение будет наблюдаться при ширине квантовой ямы $4-6 \mathrm{~nm}$.
\end{abstract}

DOI: 10.21883/PJTF.2019.10.47748.17478

В современных оптоэлектронных приборах ближнего и среднего инфракрасного диапазона при высоких значениях концентрации носителей заряда наблюдается сильное внутризонное поглощение излучения $[1,2]$. Значительный вклад в коэффициент внутризонного поглощения вносит процесс поглощения излучения свободными дырками с переходом их в спин-отщепленную (so) зону. Исследование данного процесса было проведено авторами в работе [3] для гетероструктур AlSb/InAsSb и InGaAsP/InP в приближении сферического ненапряженного $k P$-гамильтониана в восьмизонной модели Кейна $[4,5]$. Использовалась модификация [6] модели Кейна, основанная на сведении задачи на собственные функции и собственные значения $k P$-гамильтониана к решению системы дифференциальных уравнений для компонент волновых функций носителей заряда в спинорной форме. В работе [7] была решена задача для восьмизонного $k P$-гамильтониана с учетом несферичности.

В современной оптоэлектронике важную роль играют материалы на основе напряженных гетероструктур (ГС), например соединения GaAs/InGaAs и GaSb/InAs. Ocoбенностью данных ГС является значительное рассогласование слоев по параметру решетки, что приводит к существенным сдвигам краев энергетических зон. В связи с этим возникает интерес к решению описанной задачи для $k P$-гамильтониана, записанного с учетом упругих напряжений. Следует отметить, что в ряде работ [8-10] представлена методика точного численного расчета зонной структуры, в том числе и в рамках $k P$-метода, однако до сих пор актуальна задача разработки подхода, позволяющего получать именно аналитические выражения для волновых функций носителей заряда и матричных элементов оптических переходов, что облегчило бы подробное теоретическое исследование микроскопических процессов в напряженных ГС.
Цель настоящей работы состоит в получении аналитических выражений энергетических спектров и волновых функций носителей заряда в квантовой яме в приближении эффективной массы в рамках восьмизонной модели Кейна. Также целью работы является численный расчет на основе полученных выражений коэффициента поглощения света тяжелыми дырками с переходом в $s o$-зону для напряженных квантовых ям (КЯ) $\mathrm{GaAs} / \mathrm{InGaAs}$, полученных в режиме метаморфного роста в направлении (100), с учетом несферичности $k P$-гамильтониана. Используемый в работе гамильтониан представляет собой кейновский $k P$-гамильтониан $8 \times 8$ с учетом квадратичных по $k$ членов, в котором упругие напряжения учитывались согласно теории Бира-Пикуса $[11,12]$. В данном гамильтониане не были учтены релятивистские поправки первого порядка по $k$. Также в кейновских уравнениях было опущено слагаемое с тяжелой массой электронов.

Используя алгебраические преобразования, можно переписать исходный гамильтониан в виде системы уравнений для компонент спиноров с $s$ - и $p$-симметрией

$$
\begin{gathered}
\left(E_{C}-E+A_{\varepsilon}\right) u=-i P \mathbf{k} \cdot \mathbf{v}, \\
\left(E_{V}-\delta-E-\frac{\hbar^{2} k^{2}}{2 m_{0}}\left(\gamma_{1}-2 \gamma_{2}\right)+P_{\varepsilon}+Q_{\varepsilon}\right) v_{i} \\
+i \delta[\boldsymbol{\sigma} \times \mathbf{v}]_{i}-\frac{3 \hbar^{2}}{m_{0}} \gamma_{3} k_{i}(\mathbf{k} \cdot \mathbf{v})+\frac{3 \hbar^{2}}{m_{0}}\left(\gamma_{3}-\gamma_{2}\right) k_{i}^{2} v_{i}=i P k_{i} u .
\end{gathered}
$$

Здесь $и$ и $\mathbf{v}$ - компоненты спинора огибающей волновой функции с $s$ - и $p$-симметрией, $E_{C}$ и $E_{V}-$ значения энергии дна зоны проводимости и потолка валентной зоны, $P$ - кейновский матричный элемент, $\delta=\Delta_{s o} / 3$ - константа спин-орбитального расщепления, $\gamma_{1}, \gamma_{2}, \gamma_{3}$ - обобщенные параметры Латтинжера, $m_{0}-$ 

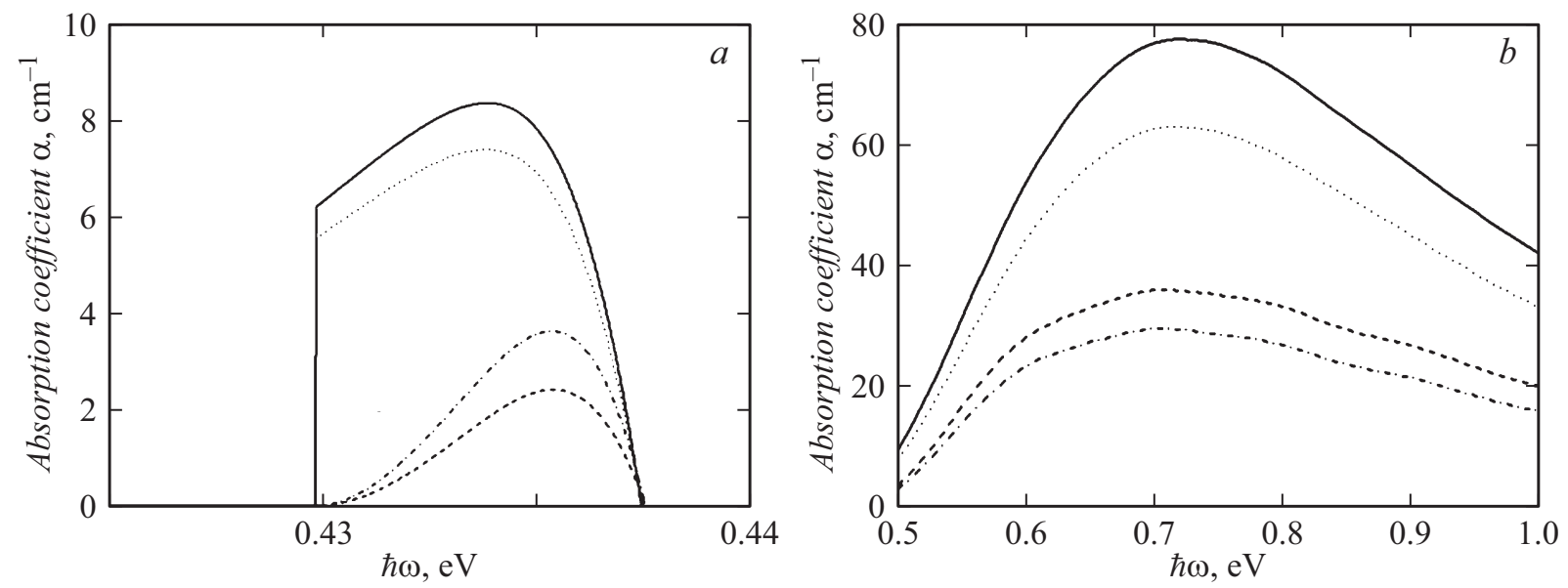

Рис. 1. Зависимость коэффициента поглощения излучения тяжелыми дырками с переходом в спектр локализованных (a) и делокализованных $(b)$ состояний so-дырок от энергии оптического перехода в гетероструктуре InGaAs/GaAs при ширине КЯ $a=5 \mathrm{~nm}$ и массовой доле индия $y=0.3$. Сплошной и пунктирной линиями показаны результаты расчета для ТЕ-поляризованной световой волны без учета и с учетом несферичности и упругих напряжений соответственно, штриховой и штрихпунктирной аналогичные результаты для ТМ-поляризованной световой волны.

масса свободного электрона, $\boldsymbol{\sigma}=\left(\sigma_{x}, \sigma_{y}, \sigma_{z}\right)$ - матрицы Паули, $E$ и $\mathbf{k}$ - энергия и волновой вектор частицы, $P_{\varepsilon}=a_{v}\left(\varepsilon_{x x}+\varepsilon_{y y}+\varepsilon_{z z}\right), Q_{\varepsilon}=\left(\varepsilon_{y y}+\varepsilon_{z z}-2 \varepsilon_{x x}\right) b / 2$, $A_{\varepsilon}=a_{c}\left(\varepsilon_{x x}+\varepsilon_{y y}+\varepsilon_{z z}\right), a_{c}, a_{v}, b$ - константы деформационного потенциала [12], $\varepsilon_{y y}=\varepsilon_{z z}=\left(a_{0}-a_{\varepsilon}\right) / a_{\varepsilon}$, $\varepsilon_{x x}=-2 C_{12} \varepsilon_{y y} / C_{11}, a_{0}$ и $a_{\varepsilon}-$ значения постоянной решетки барьера и активного слоя, $C_{11}$ и $C_{12}-$ компоненты тензора упругих модулей.

Уравнение для энергетического спектра тяжелых дырок, полученное в результате решения системы уравнений (1), имеет вид

$$
\begin{gathered}
E_{1}^{3}-C_{h} k^{2} E_{1}^{2}+\left\{C_{h}^{2}\left(k_{x}^{2} k_{y}^{2}+k_{y}^{2} k_{z}^{2}+k_{z}^{2} k_{x}^{2}\right)-3 \delta^{2}\right\} E_{1} \\
-C_{h}^{3} k_{x}^{2} k_{y}^{2} k_{z}^{2}+2 C_{h} k^{2} \delta+2 \delta^{3}=0
\end{gathered}
$$

где

$$
\begin{gathered}
E_{1}=E+\hbar^{2} k^{2} / 2 m_{h}-P_{\varepsilon}-Q_{\varepsilon}+\delta-E_{V}, \\
C_{h}=3\left(\gamma_{3}-\gamma_{2}\right) \hbar^{2} / m_{0}, \quad m_{h}=m_{0} /\left(\gamma_{1}-2 \gamma_{2}\right) .
\end{gathered}
$$

Волновые функции тяжелых дырок находятся из условия $\nabla \mathbf{v}=0$. Тогда, задавая вид функции $v_{x}$, можно однозначно найти вид компонент $v_{y}$ и $v_{z}$. Решение задачи для электронов, легких и $s o$-дырок можно получить, если выразить скалярное произведение $\mathbf{k} \cdot \mathbf{v}$ из первого уравнения системы (1) и подставить во второе. Тогда можно выразить компоненты спинора $\mathbf{v}$ через производные спинора $u$ по координатам.

Выражение для энергетического спектра носителей заряда получается при подстановке полученных волно- вых функций в первое уравнение системы (1)

$$
\begin{aligned}
(E & -E_{C}-A_{\varepsilon}+P f_{31}\left[g_{x 1} k_{y}^{2}+g_{y 1} k_{x}^{2}\right] \\
& \left.+P k_{z}^{2}\left\{f_{1}-f_{2} f_{4}\left[2 g_{t 1}-g_{x 1}-g_{y 1}\right]\right\} /\left(1+C k_{z}^{2}\right)\right)^{2} \\
& =P^{2} k_{z}^{2}\left\{f_{2} f_{31} /\left(1+C k_{z}^{2}\right)-f_{4}\right\}^{2}\left(\left(g_{t 1}-g_{y 1}\right)^{2} k_{x}^{2}\right. \\
& \left.+\left(g_{t 1}-g_{x 1}\right)^{2} k_{y}^{2}\right),
\end{aligned}
$$

где

$$
\begin{gathered}
g_{x 1}=1+C k_{x}^{2}-f_{2}^{2} /\left(1+C k_{z}^{2}\right), \\
g_{y 1}=1+C k_{y}^{2}-f_{2}^{2} /\left(1+C k_{z}^{2}\right), \\
g_{t 1}=-f_{2}-f_{2}^{2} /\left(1+C k_{z}^{2}\right), \\
f_{31}=f_{1} /\left(g_{x 1} g_{y 1}-g_{t 1}^{2}\right), \quad f_{2}=-E_{1}^{-1} \delta, \\
f_{1}=E_{1}^{-1}\left\{P+3 \gamma_{3} \hbar^{2}\left(E-E_{C}-A_{\varepsilon}\right) / m_{0} P\right\}, \\
C=-C_{h} E_{1}^{-1} .
\end{gathered}
$$

Для нахождения волновых функций носителей заряда в КЯ необходимо положить в области КЯ для тяжелых дырок $v_{x}=H_{1} \cos k_{x} x \xi+H_{2} \sin k_{x} x \eta$ (где $\xi=1 / \sqrt{2}(1-1)^{*}, \eta=1 / \sqrt{2}(11)^{*}$, звездочка обозначает комплексное сопряжение, $k_{x}$ - квантованная компонента волнового вектора, $H_{1}$ и $H_{2}-$ нормировочные коэффициенты) и $u=A_{1} \cos k_{x} x \eta+A_{2} \sin k_{x} x \xi$ (где $A_{1}$ и $A_{2}$ - нормировочные коэффициенты) для электронов, легких и $s o$-дырок.

Полученные таким образом выражения для волновых функций являются достаточно громоздкими, однако в случае $k_{z}=0$ выражения существенно упрощаются. Они совпадают с полученными в работе [7] результатами для ненапряженных ГС, в которых произведена замена $E_{C} \rightarrow E_{C}+A_{\varepsilon}, E_{V} \rightarrow E_{V}+P_{\varepsilon}+Q_{\varepsilon}$. 

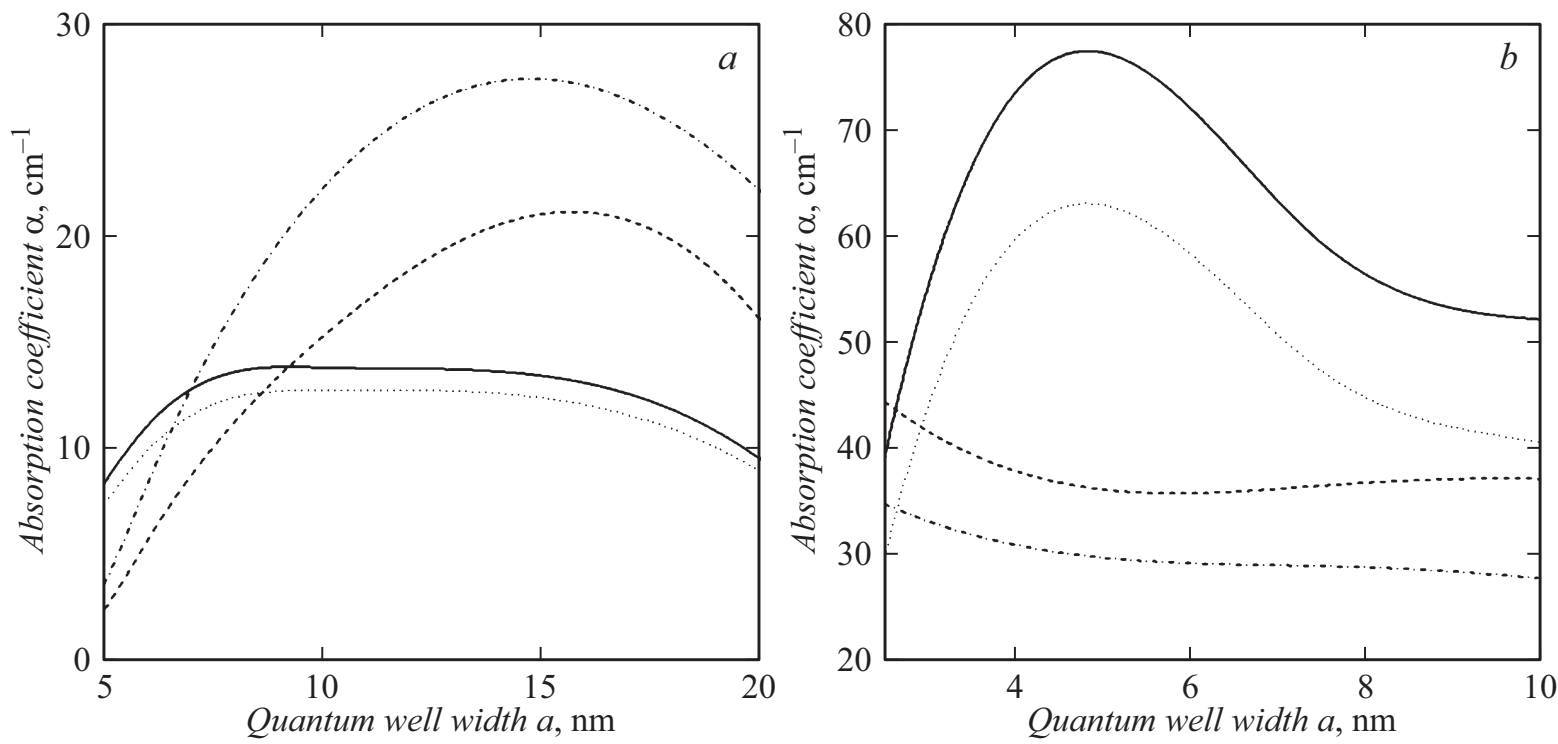

Рис. 2. Зависимость максимального значения коэффициента поглощения излучения тяжелыми дырками с переходом в спектр локализованных $(a)$ и делокализованных $(b)$ состояний so-дырок в гетероструктуре InGaAs/GaAs от ширины КЯ при массовой доле индия $y=0.3$. Обозначения кривых соответствуют принятым на рис. 1 .

Для нахождения уровней размерного квантования необходимо решить систему уравнений (1) независимо для области КЯ и области барьера и сшить полученные решения на гетерогранице с использованием граничных условий, которые могут быть выведены с помощью процедуры интегрирования кейновских уравнений через интерфейс $[5,6,13]$. В общем виде они имеют достаточно громоздкий вид, однако практически без потери точности можно использовать соотношения

$$
\begin{aligned}
& \left(v_{x}\right)_{-}^{+}=0, \quad\left(v_{y}\right)_{-}^{+}=0, \quad\left(\partial v_{y} / \partial x\right)_{-}^{+}=0, \\
& \left(\hbar^{2}\left(\gamma_{1}+4 \gamma_{2}-6 \gamma_{3}\right)\left(\partial v_{x} / \partial x\right)\right)_{-}^{+}=\left(-2 m_{0} P u\right)_{-}^{+} .
\end{aligned}
$$

Далее необходимо рассматривать дырочную волновую функцию как суперпозицию состояний тяжелых, легких и so-дырок. Поскольку константа спин-орбитального взаимодействия достаточно велика, можно рассматривать $s o$-дырки и суперпозицию легких и тяжелых дырок по отдельности. Для обоих случаев получается система уравнений для нормировочных коэффициентов, характеристическое уравнение которой представляет собой дисперсионное уравнение для нахождения уровней размерного квантования. Для нахождения окончательного выражения для нормировочных коэффициентов необходимо также применить условие нормировки в потенциальном ,ящике“.

Для расчета коэффициента поглощения необходимо выполнить расчет матричных элементов оптических переходов и затем произвести суммирование по всем начальным и конечным состояниям. В общем виде данная задача является довольно сложной вследствие весьма громоздкого вида точных волновых функций. Для упрощения задачи можно использовать приближение, в соответствии с которым свойства системы инвариантны относительно поворота вокруг оси роста кристалла (грубо говоря, считать, что для носителей заряда $\left.m_{y}=m_{z} \neq m_{x}\right)$. В этом случае можно направить ось $y$ вдоль компоненты $q$ волнового вектора в плоскости КЯ, тогда компонента $k_{z}=0, k_{y}=q$ и для расчета коэффициента поглощения можно использовать метод, описанный в работе [3]. В итоге для коэффициента поглощения излучения тяжелыми дырками с переходом в $s o$-зону в расчете на одну яму можно получить

$$
\alpha_{p}^{d}=\frac{2 P^{2} e^{2}}{\hbar^{2} c} \frac{1}{n \omega a} \sum_{n_{h}} \sum_{n_{s o}} m_{r}(q) \sigma_{p}^{d}(q) f_{h}(q)
$$

для переходов в спектр локализованных состояний so-дырок и

$$
\alpha_{p}^{c}=\frac{P^{2} e^{2}}{\pi \hbar^{2} c} \frac{1}{n \omega a} \sum_{n_{h}} \int_{0}^{q_{\max }(\hbar \omega)} \frac{m_{r}(q) \sigma_{p}^{c}(q) f_{h}(q) q}{k_{s o}(q)} d q
$$

для переходов в спектр делокализованных состояний so-дырок.

Здесь $n-$ показатель преломления, $n_{h}$ и $n_{s o}-$ номера подзон тяжелых и $s o$-дырок, $\omega$ - частота оптического перехода, $a-$ ширина КЯ, $k_{s o}-x$-компонента волнового вектора $s o$-дырки, $f_{h}(q)-$ функция распределения тяжелых дырок, $m_{r}(q)=m_{h h}(q) m_{s o}(q) /\left|m_{h h}(q)-m_{s o}(q)\right|-$ величина, аналогичная приведенной массе, $m_{h h}(q)$ и $m_{s o}(q)$ - зависящие от $q$ эффективные массы тяжелой и $s o$-дырки, индекс $p$ определяет поляризацию света. Величина $\sigma_{p}$ определяется интегралами перекрытия волновых функций. При распространении света в плоскости КЯ: $\sigma_{\mathrm{TM}}=2 \pi\left|I_{x}^{j, a}\right|^{2}, \sigma_{\mathrm{TE}}=\pi\left(\left|I_{y}^{j, s}\right|^{2}+\left|I_{z}^{j, s}\right|^{2}\right)$, где 
$I_{i}=\int_{-a / 2}^{a / 2} v_{h, i}^{*} u_{s o} d x$. В силу монотонности зависимости $\hbar \omega(q)$ из (5) и (6) можно численно получить зависимости $\alpha_{p}^{d, c}(\hbar \omega)$.

В настоящей работе при всех расчетах были приняты значения температуры $300 \mathrm{~K}$ и концентрации дырок $10^{12} \mathrm{~cm}^{-2}$. Для расчета сдвигов краев зон был использован метод, описанный в работе [14]. Расчет показывает, что глубина потенциальной ямы для легких и $s o$-дырок становится достаточно малой. Более того, при значениях массовой доли индия $y<0.15$ будет наблюдаться барьер для легких дырок, а при $y>0.55$ - для спинотщепленных.

Результаты численного расчета коэффициента поглощения представлены на рис. 1 и 2. На рис. 1 показана зависимость коэффициента поглощения света тяжелыми дырками с переходом в спектр локализованных $(a)$ и делокализованных $(b)$ состояний so-дырок от энергии оптического перехода. Из рисунков видно, что в отличие от аналогичных результатов для ГС $\mathrm{AlSb} / \mathrm{InAsSb}$ и InGaAsP/InP [3] поглощение с переходом в спектр делокализованных состояний so-дырок является более сильным, чем поглощение с переходом в спектр локализованных состояний. Данный факт можно объяснить тем, что для $\mathrm{InGaAs} / \mathrm{GaAs}$ яма для so-дырок является очень мелкой и они локализованы слабо в отличие от ГС $\mathrm{AlSb} / \mathrm{InAsSb}$ и $\mathrm{InGaAsP} / \mathrm{InP}$, где яма для so-дырок является достаточно глубокой. Также видно, что для обоих случаев коэффициент поглощения для ТЕ-поляризованной волны значительно больше, чем для ТМ-поляризованной. Учет упругих напряжений и несферичности приводит к уменьшению поглощения с переходом в спектр делокализованных состояний so-дырок для обеих поляризаций падающего излучения. Для переходов в спектр локализованных состояний учет упругих напряжений и несферичности приводит к увеличению коэффициента поглощения для ТМ-поляризации и уменьшению для ТЕ-поляризации.

На рис. 2 показана зависимость максимального значения коэффициента поглощения от ширины КЯ для переходов в спектр локализованных $(a)$ и делокализованных $(b)$ состояний $s o$-дырок. Видно, что коэффициент поглощения с переходом в спектр локализованных состояний $s o$-дырок имеет максимум в районе $a=8 \mathrm{~nm}$ для ТЕ-поляризованной световой волны и $a=15 \mathrm{~nm}$ для ТМ-поляризованной. Столь большое значение ширины КЯ, соответствующей максимальному поглощению, объясняется тем, что яма для so-дырок является очень мелкой, и только при достаточно большой ширине КЯ носители заряда будут сильно локализованы. Для поглощения с переходом в спектр делокализованных состояний максимальное поглощение будет наблюдаться при $a=5 \mathrm{~nm}$, так как яма для тяжелых дырок является достаточно глубокой и они хорошо локализованы даже при малых значениях ширины КЯ.

\section{Благодарности}

Авторы выражают благодарность В.Я. Алёшкину (Институт физики микроструктур РАН) за участие в обсуждении научных результатов.

\section{Финансирование работы}

Работа выполнена при поддержке Российского фонда фундаментальных исследований (грант № 17-32-50145).

\section{Список литературы}

[1] Zory P.S. Quantum well lasers. N.Y.: Academic Press, 1993. $504 \mathrm{p}$.

[2] Шик А.Я. // Письма в ЖТФ. 1989. Т. 15. В. 8. С. 40-43.

[3] Павлов Н.В., Зегря А.Г., Зегря Г.Г., Бугров В.Е. // ФТП. 2018. T. 52. B. 2. C. 207-220.

[4] Kane E.O. // J. Phys. Chem. Solids. 1957. V. 1. N 4. P. 249 261.

[5] Bastard G. // Wave mechanics applied to semiconductor heterostructures. N.Y.: John Wiley and Sons, Inc., 1990. 357 p.

[6] Polkovnikov A.S., Zegrya G.G. // Phys. Rev. B. 1998. V. 58. N 7. P. 4039-4056.

[7] Pavlov N.V., Zegrya G.G. // J. Phys.: Conf. Ser. 2018. V. 993. P. 012013.

[8] Szmulowicz F., Haugan H., Brown G.J. // Phys. Rev. B. 2004. V. 69. N 15. P. 155321.

[9] Глинский Г.Ф., Миронова М.С. // ФТП. 2014. Т. 48. В. 10. C. $1359-1369$.

[10] Глинский Г.Ф. // Письма в ЖТФ. 2018. Т. 44. В. 6. С. 17-24.

[11] Liu G., Chuang S.L. // Phys. Rev. B. 2002. V. 65. N 16. P. 165220.

[12] Бир Г.Л., Пикус Г.Е. Симметрия и деформационные эффекты в полупроводниках. М.: Наука, 1972. 584 с.

[13] Burt M.G. // J. Phys.: Condens. Matter. 1992. V. 4. N 32. P. 6651-6690.

[14] Krijn M.P.C.M. // Semicond. Sci. Technol. 1991. V. 6. N 1. P. 27-31. 